\title{
What is pressure? Evidence for social pressure as a type of regulatory focus
}

\author{
Darrell A. Worthy, Arthur B. Markman, and W. Todd Maddox \\ University of Texas, Austin, Texas
}

\begin{abstract}
Previous research (Markman, Maddox, \& Worthy, 2006) suggests that pressure leads to choking when one is learning to classify items on the basis of an explicit rule, but it leads to excelling when one is learning to classify items on the basis of an implicit strategy. In this article, we relate social pressure to regulatory focus theory. We propose that the effects of pressure on performance arise because pressure induces a prevention focus that interacts with the more local reward structure of the task. To test this hypothesis, we repeated previous research, but using a losses reward structure, so that participants under pressure were in a regulatory fit. We also successfully replicated previous results by using a gains reward structure. In contrast with participants who attempted to maximize gains on each trial, participants who attempted to minimize losses choked on the implicit-learning task but excelled on the explicit-learning task. The results suggest a three-way interaction between pressure level, task type, and reward structure.
\end{abstract}

Pressure affects performance on several cognitive and motor tasks (see, e.g., Beilock \& Carr, 2001, 2005; Beilock \& DeCaro, 2007; Beilock, Kulp, Holt, \& Carr, 2004; Markman, Maddox, \& Worthy, 2006; Masters, 1992). In these tasks, participants often choke under pressure, underperforming on a task relative to their normal performance level because of an acute stressor. Intriguingly, participants can also excel under pressure, performing better than they would otherwise perform without pressure. For example, Markman et al. (2006) found that participants choked under pressure when performing a classification task that required an explicit rule-based strategy, but excelled under pressure when performing a task that required an implicit information-integration strategy.

These results are consistent with the distraction hypothesis (Beilock \& Carr, 2005; Beilock et al., 2004; Markman et al., 2006; Wine, 1971), which proposes that pressure decreases available executive-attention or working memory resources, leading to performance decrements on tasks that require an explicit strategy. A decline in available executive-attention resources also increases people's reliance on implicit strategies, which enhances performance on implicit information-integration tasks.

\section{Relating Pressure to Regulatory Focus}

In this article, we explore the possibility, raised by Markman et al. (2006), that pressure manipulations induce a situational regulatory focus in participants (Grimm, Markman, Maddox, \& Baldwin, 2008; Higgins, 1997; Maddox, Baldwin, \& Markman, 2006; Markman, Baldwin, \& Maddox, 2005; Worthy, Maddox, \& Markman, 2007). Regulatory focus theory posits that people adopt one of two distinct regulatory foci: a promotion focus, in which one becomes sensitive to potential gains in the environment, or a prevention focus, in which one becomes sensitive to potential losses in the environment. The pressure manipulation that has been used in a number of studies has hinged on the potential for a negative social outcome if the participant lets down a partner (see, e.g., Beilock \& Carr, 2001; Beilock et al., 2004; Gray, 2004; Markman et al., 2006). This social pressure may induce a prevention focus. In contrast, participants who are not given a pressure manipulation are more likely to have a promotion focus, because they are simply trying to complete the experiment and earn the required credit for participation.

Previous research has suggested that a person's global regulatory focus interacts with the more local gains or losses that are available on each trial of the task that is being performed (Higgins, 2000; Maddox et al., 2006; Worthy et al., 2007). When there is a fit between regulatory focus and reward structure (i.e., a global promotion focus with gains, or a global prevention focus with losses), people are more capable of solving complex problem-solving tasks that require executive attention than they are when there is a mismatch (i.e., a global prevention focus with gains, or a global promotion focus with losses; Grimm et al., 2008; Maddox et al., 2006; Markman et al., 2005; Worthy et al., 2007). Previous research has found that participants in a regulatory fit perform explicit rule-based learning tasks better than those in a mismatch, but do worse in implicit information-integration-learning tasks (Grimm et al., 2008; Maddox et al., 2006; Markman et al., 2005). There is also evidence that increased working memory capacity may enhance performance on rule-based tasks but hinder performance on information-integration tasks (see, e.g., DeCaro, Thomas, \& Beilock, 2008; Tharp \& Pickering,

D. A.Worthy, worthyda@mail.utexas.edu 
in press). One explanation for the improved performance on explicit rule-based tasks for participants in a regulatory fit is that a fit leads to an increase in executive working memory resources.

The results from Markman et al. (2006) are thus consistent with both the distraction hypothesis and the regulatory fit hypothesis. In the present article, we test the differences between the two hypotheses by replicating the previous study, but with a losses reward structure. In a losses reward structure, participants lose points throughout the task and must minimize their losses. According to the regulatory fit hypothesis, which adds an intermediate step to the distraction hypothesis, pressure does not decrease available working memory directly; rather, it induces a prevention focus, which interacts with the reward structure of the task to create either a regulatory fit or a regulatory mismatch. The regulatory mismatch causes decrements in available executive resources.

We thus propose that participants who perform a task under low pressure have different regulatory foci from those of participants who perform under high pressure. Those who perform under low pressure have a situational promotion focus, whereas those who perform under high pressure have a situational prevention focus. These distinct foci interact with the local reward structure to produce either a regulatory fit or a regulatory mismatch. Participants who are in a pressure-induced prevention focus and are performing a task in which responses result in losses would be in a regulatory fit; thus, these participants would have an increased amount of available executive-attention resources. Those who perform rule-based tasks would now excel under pressure, whereas those who perform information-integration tasks would choke. The distraction hypothesis predicts the opposite, because it proposes that pressure leads directly to decrements in available executive-attention resources, regardless of the reward structure of the task. We test these two hypotheses in the present experiment.

\section{EXPERIMENT 1}

In Experiment 1, participants learned to classify stimuli into one of two categories. Half of the participants classified stimuli from a rule-based category structure. Figure 1A depicts a simple rule-based task in which the participant focused on one of two dimensions and determined the location of the stimuli on the basis of the dimension that separated the two categories. This type of task is typically thought to involve explicit hypothesis testing, in which case performance would be harmed by any procedure that decreases executive-attention resources (Ashby, AlfonsoReese, Turken, \& Waldron, 1998; Maddox \& Ashby, 2004; Maddox, Filoteo, Hejl, \& Ing, 2004).

The other participants classified stimuli using an information-integration category structure. The stimulus structure in Figure 1B rotates the category boundary $45^{\circ}$ in stimulus space; thus, the rule that separates the categories cannot be stated easily. This stimulus configuration is thought to be learned best by a procedural or similaritybased process that is limited in its demands on working memory (Maddox \& Ashby, 2004; Maddox, Ashby, \&
A

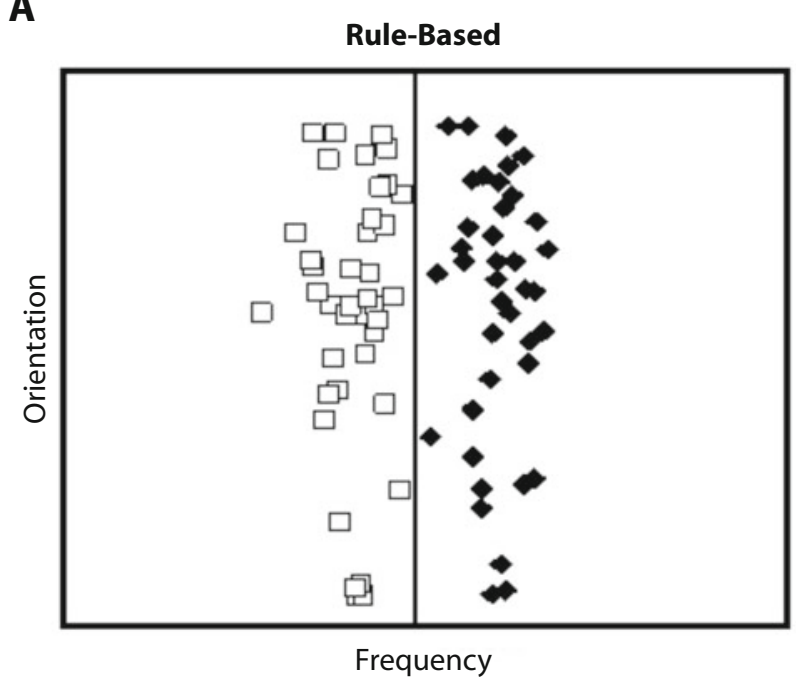

B

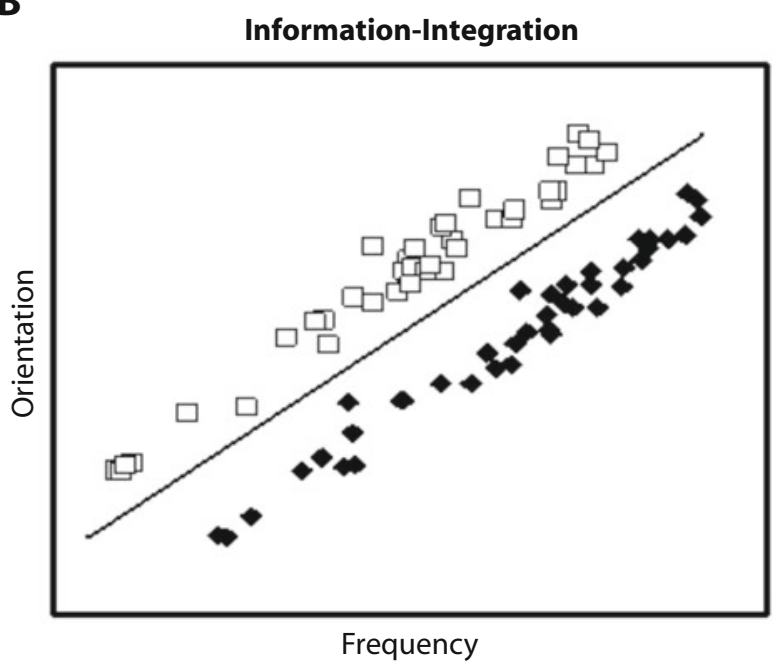

Figure 1. Two category structures that were used in Experiments 1 and 2: a unidimensional rule-based structure (A), and a two-dimensional information-integration structure (B).

Bohil, 2003). Explicit hypothesis-testing strategies can be used to solve these tasks, but they lead to suboptimal performance.

In the present experiment, participants lost points on each trial, but they lost fewer points for a correct classification than for an incorrect one. Their goal was to minimize the number of points that they lost. Participants were placed under either low or high pressure. Those who were under low pressure were simply asked to do their best by trying to lose as few points as possible. Participants who were under high pressure were told that they could earn a monetary bonus if both they and a (fictional) partner lost no more than a certain number of points. They were informed, furthermore, that their partner had already performed the task and reached the criterion; thus, it was up to the participants to earn the bonus for themselves and their partners. This type of manipulation has been used in 
numerous previous studies and has been shown to induce pressure (see, e.g., Beilock \& Carr, 2001, 2005; Beilock \& DeCaro, 2007; Beilock et al., 2004; Gray, 2004). ${ }^{1}$

\section{Method}

The participants were 80 members of the University of Texas community, and they received course credit for their participation. Participants were randomly assigned to one of four between-subjects conditions, which consisted of the factorial combination of two category types (rule based vs. information integration) and two types of pressure (high vs. low). One participant in the information-integration low-pressure condition was excluded because of experimenter error.

Participants in the low-pressure condition were asked to do their best. Participants in the high-pressure condition were told that they could earn a monetary bonus (\$6) if both they and a (fictional) partner achieved a performance criterion ( $80 \%$ correct) over the final 80 -trial block of the experiment. They were then informed that their partner had already completed the experiment and reached the performance criterion, so the partner was relying on the participant to earn both of them the bonus.

The stimuli were Gabor patches that varied in frequency (spacing of the bars) and orientation relative to the computer screen. Participants performed eight blocks of 80 trials. In each trial, a stimulus was presented on the screen, and participants were asked to indicate which category they thought the stimulus belonged to by pressing a key. They were then given corrective feedback. Participants under high pressure also heard a "ching" or buzzer sound as feedback for correct or incorrect classifications, respectively, on each trial. Participants were told that they would lose points on each trial and that their goal was to minimize their losses. The participants lost only 1 point for a correct response, but they lost 3 points for an incorrect response. A meter on the right-hand side of the screen indicated how many points had been lost, and a line indicated how many points had to remain for reception of the bonus. The meter was reset before the beginning of each block.

\section{Results}

Performance measures. Figure 2 shows the mean accuracy for each condition in each block. The data were subjected to a 2 (pressure level) $\times 2$ (category type) $\times$ 8 (block) ANOVA. There was a significant category type $\times$ pressure level interaction $[F(1,75)=5.32, p<$ $\left..05, \eta^{2}=.07\right]$. To examine the nature of the interaction, we compared the performance of participants in the lowand high-pressure conditions within each category structure. In direct contrast with the data from Markman et al.
(2006), participants in the rule-based high-pressure condition outperformed participants in the rule-based lowpressure condition during every block of the experiment $(p<.01$ by sign test). Also in contrast with Markman et al.'s (2006) findings, participants in the informationintegration low-pressure condition outperformed those in the information-integration high-pressure condition on each block ( $p<.01$ by sign test).

Model-based analyses. One advantage to using simple stimuli is that we can fit mathematical models to each participant's data to determine what strategy they used to solve the task. We fit decision-bound models to the data from individual participants on a block-by-block basis (Maddox, 1999; Maddox \& Ashby, 1993). Decisionbound models assume that participants used a decision bound to separate stimuli into two categories, with the stimuli that are on one side of the bound being classified into one category, and the stimuli that are on the other side of the bound being classified into the other category. The optimal decision bound in the rule-based condition is depicted by the vertical line in Figure 1A. The optimal decision bound in the information-integration condition is depicted by the diagonal line in Figure 1B.

Four models were fit. One was a (two-parameter) rule-based model that assumed a unidimensional decision bound along the spatial-frequency dimension (with another variant that assumed a decision bound along the spatial orientation dimension). The location of the decision bound was a free parameter, as well as a "noise" parameter that represented variability in the trial-by-trial memory for, and application of, the decision bound. The second model was a (three-parameter) rule-based model that assumed a conjunctive strategy. This model assumed one decision boundary along spatial frequency and a second along spatial orientation, and it assumed the decision rule: Respond " $\mathrm{A}$ " if the spatial frequency is low and the orientation is steep; otherwise, respond "B." A second variant assumed a different rule: Respond " $\mathrm{B}$ " if the spatial frequency is high and the orientation is low; otherwise, respond "A." The third model was a (threeparameter) information-integration model that assumed a linear decision boundary. The slope and the intercept

\section{Accuracy by Block}

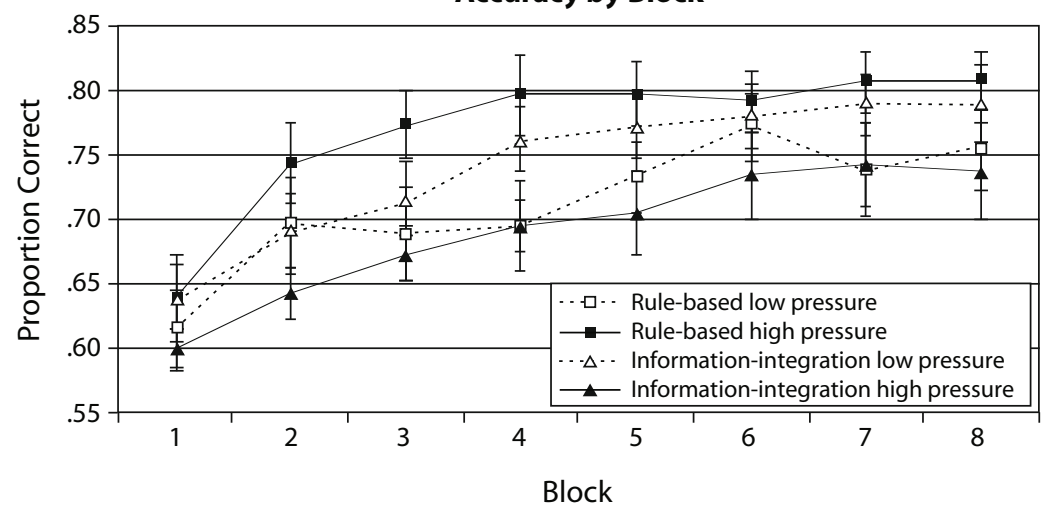

Figure 2. Mean accuracy for the two category structures in the low- and highpressure conditions from Experiment 1. 
of the linear decision boundary were free parameters, as well as the "noise" parameter that was outlined above. The fourth model was a (one-parameter) guessing model that assumed that the probability of responding "A" (a free parameter in the model) was not affected by the location of the stimulus in space. The Akaike information criterion (AIC; Akaike, 1974) was used to determine the model that provided the best account of the data. AIC penalizes a model for each free parameter; thus, a model with fewer parameters can provide a better account of the data than a model with more free parameters can.

Figure $3 \mathrm{~A}$ displays the proportion of rule-based participants best fit by a unidimensional-rule model on the frequency dimension (i.e., the optimal model). A higher proportion of high-pressure participants were best fit by a rule-based model in six of eight blocks, although the difference was not significant. Most participants whose data were not fit best by the unidimensional rule-based model on the frequency dimension were better fit by the guessing model or an information-integration model. Figure 3B shows the proportion of information-integration participants whose data were fit best by an information-integration model. A higher proportion of data sets from low-pressure participants in the information-integration condition were fit best by an information-integration model in all eight blocks $(p<.05$ by sign test). Data that were not fit best by an information-integration model were usually fit better by a conjunctive or unidimensional rule-based model.

Our process-based account, whereby a regulatory fit leads to an increase in executive resources, suggested that overall rule use should have been higher for participants who were in the pressure conditions and performed both tasks (i.e., the proportion of data sets should be fit best by either one of the two unidimensional rule models or the conjunctive model). This is exactly what we found when we examined the proportion of participants in each block who were fit by any of the three rule models (unidimensional frequency, unidimensional orientation, and conjunctive). More participants performing rule-based tasks under high pressure were best fit by rule models in seven of eight blocks of the experiment ( $p<.01$ by sign test) than participants performing rule-based tasks under low pressure, and more participants performing information-integration tasks under high pressure were best fit by rule models in

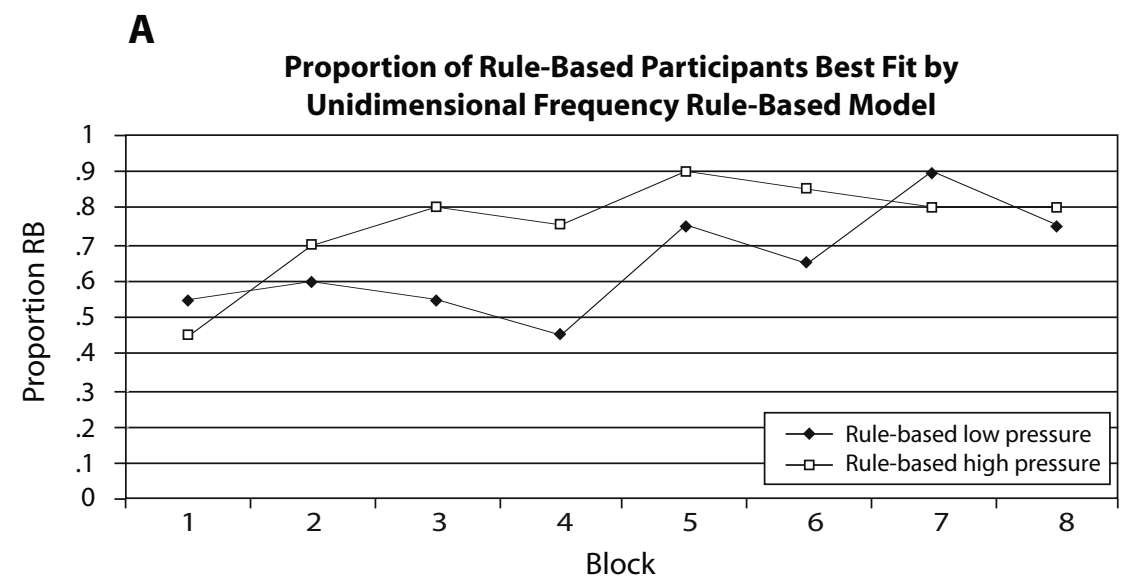

B

Proportion of Information-Integration Participants Best Fit by Information-Integration Model

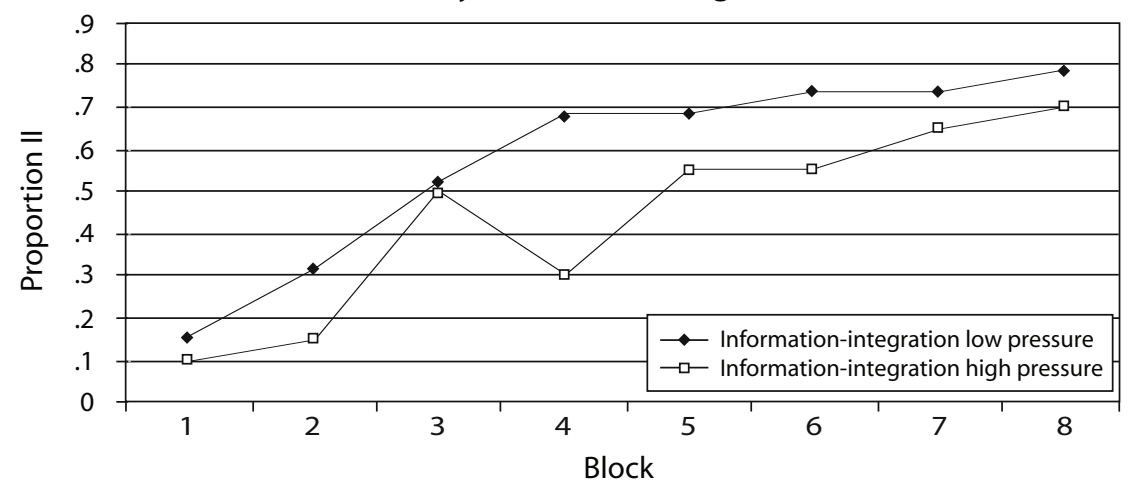

Figure 3. Model results from Experiment 1. (A) Proportion of participants learning rulebased (RB) categories who were best fit by a unidimensional rule-based model on the spatialfrequency dimension. (B) Proportion of participants learning information-integration (II) categories who were best fit by an information-integration model. 
all eight blocks of the experiment than participants performing information-integration tasks under low pressure. The evidence of greater use of explicit rules indicates that, when one is given a task with a losses reward structure, pressure causes an increase in executive resources, as predicted by regulatory focus theory.

\section{EXPERIMENT 2}

To ensure that these results did not differ from Markman et al.'s (2006) results because of a difference in the time at which the studies were conducted, we replicated Markman et al.'s (2006) original experiment, which used a gains reward structure.

\section{Method}

An additional 40 participants were randomly placed into one of four between-subjects conditions that consisted of the factorial combination of two category types (rule based vs. information integration) and two types of pressure (high vs. low). Unlike in Experiment 1 , these participants gained 2 points for each correct classification and earned 0 points for each incorrect classification. The remainder of the method, including the pressure manipulation, was the same as that in Experiment 1.

\section{Results}

We obtained the same pattern of results as that of Markman et al. (2006). The data were subjected to a 2 (pressure level) $\times 2$ (category type) $\times 8$ (block) ANOVA. There was a significant category type $\times$ pressure level interaction $\left[F(1,36)=7.74, p<.01, \eta^{2}=.18\right]$. Figure 4 shows the accuracies averaged across blocks for participants in each of the eight conditions across Experiments 1 and 2. In contrast with the results of Experiment 1, participants who performed rule-based tasks in Experiment 2 choked under pressure, and participants who performed informationintegration tasks excelled under pressure, when they were gaining points for correct responses.

To examine the effect of altering the reward structure, we conducted a 2 (reward structure $) \times 2$ (pressure level) $\times$ 2 (category type) $\times 8$ (block) repeated measures ANOVA using the combined data from Experiments 1 and 2. There was a significant reward structure $\times$ pressure level $\times$ cat-

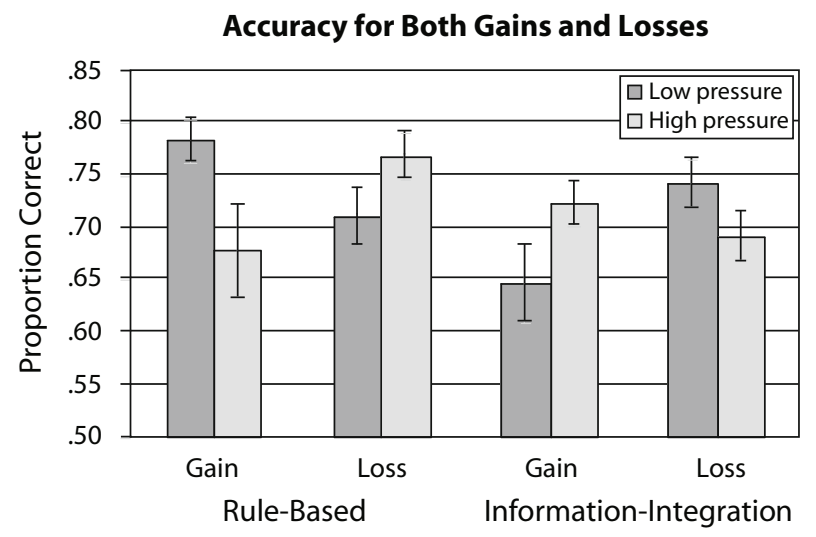

Figure 4. Mean accuracy for participants in each condition in Experiments 1 and 2, averaged across blocks. egory type interaction $\left[F(1,111)=12.84, p<.01, \eta^{2}=\right.$ .10]. When participants gained points on each trial, those who performed a rule-based task choked under pressure, and those who performed an information-integration task excelled under pressure. In contrast, when participants lost points on each trial, those who performed a rule-based task excelled under pressure, whereas those who performed an information-integration task choked under pressure.

\section{DISCUSSION}

The combined data from Experiments 1 and 2 offer clear support for the view that social pressure induces a situational prevention focus. Pressure alone did not reduce the amount of available working memory resources, contrary to the prediction of the distraction hypothesis. Instead, pressure induced a situational prevention focus that interacted with the reward structure of the task to influence the regulatory fit of participants in each condition. The results also support our hypothesis that participants in the low-pressure conditions would have a situational promotion focus (as opposed to a prevention focus or no motivational focus at all). These participants were attempting to do their best in order to earn course credit or monetary compensation for their participation. This result is consistent with a situational promotion focus. The conclusion that participants in our low-pressure conditions were in a promotion focus is supported by differences in performance for low-pressure-condition participants that were based on the reward structure of the task.

The different foci for participants under different levels of pressure led to different interactions with the local reward structures of the tasks. When participants were in a regulatory fit (low-pressure condition and receiving gains, or high-pressure condition and receiving losses), they performed well on rule-based tasks but poorly on information-integration tasks. In contrast, when participants were in a regulatory mismatch (low-pressure condition and receiving losses, or high-pressure condition and receiving gains), they performed well on informationintegration tasks but poorly on rule-based tasks.

One key question is why a regulatory fit leads to an increase in executive resources. Much work to date has suggested that a regulatory fit leads to an increased experience of "feeling right," which leads to engagement in reactions and increased confidence in performance (see, e.g., Aaker \& Lee, 2006; Higgins, 2000). This increased engagement and confidence in performance may increase executiveattention resources. In contrast, a regulatory mismatch may lead to a decreased sense of "feeling right," which may produce the type of worry or anxiety about one's performance that can decrease executive-attention resources.

From a processing standpoint, it is important to point out that the distraction hypothesis is still correct when a task has a gains reward structure. Pressure still causes decrements as a result of the co-option of the working memory that is available for the task. Here we are simply extending the theory by showing that the workingmemory "distraction" is caused by an interaction between the pressure-induced situational prevention focus and the 
more local reward structure of the task. Pressure does not simply cause a reduction in executive resources; it alters one's motivational state, which then interacts with the reward structure of the environment. Although it may seem counterintuitive to suggest that heightened pressure can actually increase executive resources, anecdotal accounts of excelling under pressure are perhaps as ubiquitous as those of choking under pressure (see, e.g., Worthy, Markman, \& Maddox, in press). In this article, we have presented experimental evidence for both choking and excelling, and we have proposed an intriguing explanation for the existence of both phenomena.

The present study demonstrates the need for a broader view of the effects of pressure on performance. Most laboratory tasks are conducted using a reward matrix in which participants gain points on each trial. Not all real-world tasks involve maximizing gains, however. For example, academic test-taking situations may be viewed by the test taker as a situation in which he or she attempts to get either as few problems wrong as possible, or as many problems correct as possible. Our results suggest that test takers who perform a task that involves explicit cognitive processing will perform better under pressure when they view the test as a task in which they avoid giving incorrect answers, because they will be in a regulatory fit. Analogously, our results suggest that test takers who perform a task that involves implicit cognitive processing will perform better under pressure when they view the test as a task in which they attempt to maximize the number of correct answers, because they will be in a regulatory mismatch. Future research examining the effects of pressure on performance should take account of the three-way interaction between pressure, task type, and reward structure to develop a fuller understanding of phenomena that involve choking and excelling.

\section{AUTHOR NOTE}

This research was supported by AFOSR Grant FA9550-06-1-0204, NIMH Grant MH077708 to W.T.M. and A.B.M., and a supplement to NIMH Grant MH077708 to D.A.W. We thank J. Scott Lauritzen, Bo Zhu, and all the research assistants in MaddoxLab for help in collecting the data. Correspondence concerning this article should be addressed to D. A. Worthy, Department of Psychology, University of Texas, 1 University Station, A8000, Austin, TX 78712 (e-mail: worthyda@mail.utexas.edu).

\section{REFERENCES}

AAKeR, J. L., \& LEE, A. Y. (2006). Understanding regulatory fit. Journal of Marketing Research, 43, 15-19.

AKAIKE, H. (1974). A new look at the statistical model identification. IEEE Transactions on Automatic Control, 19, 716-723.

Ashby, F. G., Alfonso-Reese, L. A., Turken, A. U., \& Waldron, E. M. (1998). A neuropsychological theory of multiple systems in category learning. Psychological Review, 105, 442-481.

BeILOCK, S. L., \& CARR, T. H. (2001). On the fragility of skilled performance: What governs choking under pressure? Journal of Experimental Psychology: General, 130, 701-725.

BeILOCK, S. L., \& CARR, T. H. (2005). When high-powered people fail: Working memory and "choking under pressure" in math. Psychological Science, 16, 101-105.

BeIlock, S. L., \& DeCaro, M. S. (2007). From poor performance to success under stress: Working memory, strategy selection, and mathematical problem solving under pressure. Journal of Experimental Psychology: Learning, Memory, \& Cognition, 33, 983-998.

Beilock, S. L., Kulp, C. A., Holt, L. E., \& CARR, T. H. (2004). More on the fragility of performance: Choking under pressure in mathematical problem solving. Journal of Experimental Psychology: General, 133, 584-600

DeCaro, M. S., Thomas, R. D., \& Beilock, S. L. (2008). Individual differences in category learning: Sometimes less working memory capacity is better than more. Cognition, 107, 284-294.

GraY, R. (2004). Attending to the execution of a complex sensorimotor skill: Expertise differences, choking, and slumps. Journal of Experimental Psychology: Applied, 10, 42-54.

Grimm, L. R., Markman, A. B., Maddox, W. T., \& Baldwin, G. C. (2008). Differential effects of regulatory fit on category learning. Journal of Experimental Social Psychology, 44, 920-927.

Higgins, E. T. (1997). Beyond pleasure and pain. American Psychologist, 52, 1280-1300.

HigGins, E. T. (2000). Making a good decision: Value from fit. American Psychologist, 55, 1217-1230.

Maddox, W. T. (1999). On the dangers of averaging across observers when comparing decision bound models and generalized context models of categorization. Perception \& Psychophysics, 61, 354-374.

Maddox, W. T., \& Ashby, F. G. (1993). Comparing decision bound and exemplar models of categorization. Perception \& Psychophysics, 53, 49-70

Maddox, W. T., \& Ashby, F. G. (2004). Dissociating explicit and procedural-learning based systems of perceptual category learning. Behavioural Processes, 66, 309-332.

Maddox, W. T., Ashby, F. G., \& Bohil, C. J. (2003). Delayed feedback effects on rule-based and information-integration category learning. Journal of Experimental Psychology: Learning, Memory, \& Cognition, 29, 650-662.

Maddox, W. T., Baldwin, G. C., \& Markman, A. B. (2006). A test of the regulatory fit hypothesis in perceptual classification learning. Memory \& Cognition, 34, 1377-1397.

Maddox, W. T., Filoteo, J. V., HeJl, K. D., \& Ing, A. D. (2004). Category number impacts rule-based but not information-integration category learning: Further evidence for dissociable category-learning systems. Journal of Experimental Psychology: Learning, Memory, \& Cognition, 30, 227-245.

Markman, A. B., Baldwin, G. C., \& Maddox, W. T. (2005). The interaction of payoff structure and regulatory focus in classification. Psychological Science, 16, 852-855.

Markman, A. B., Maddox, W. T., \& Worthy, D. A. (2006). Choking and excelling under pressure. Psychological Science, 17, 944-948.

Masters, R. S. W. (1992). Knowledge, knerves, and know-how: The role of explicit versus implicit knowledge in the breakdown of a complex motor skill under pressure. British Journal of Psychology, 83, 343-358.

Tharp, I. J., \& Pickering, A. D. (in press). A note on DeCaro, Thomas, and Beilock (2008): Further data demonstrate complexities in the assessment of information-integration category learning. Cognition.

Wine, J. (1971). Test anxiety and direction of attention. Psychological Bulletin, 76, 92-104.

Worthy, D. A., Maddox, W. T., \& Markman, A. B. (2007). Regulatory fit effects in a choice task. Psychonomic Bulletin \& Review, 14, 1125-1132.

Worthy, D. A., Markman, A. B., \& Maddox, W. T. (in press). Choking and excelling at the free throw line. International Journal of Creativity \& Problem Solving.

\section{NOTE}

1. Note that participants who received the pressure manipulation in some of the studies that are cited were videotaped so that their performance could be evaluated by "experts" (see, e.g., Beilock \& Carr, 2005; Beilock \& DeCaro, 2007; Beilock et al., 2004). This was not part of our manipulation, because (1) it was not part of our manipulation in our previous study (Markman et al., 2006), and (2) we were worried about participants' potential skepticism regarding the existence of "experts" in category learning. Future studies should address whether differences in the pressure manipulations that were employed affect performance in different ways.

(Manuscript received August 11, 2008; revision accepted for publication October 29, 2008.) 